\title{
Evaluation of Modeling Techniques for Agent-Based Systems
}

\author{
Onn Shehory \\ IBM Research Lab in Haifa - the Tel-Aviv site \\ IBM Building, Tel Aviv 61336, ISRAEL \\ onn@il.ibm.com
}

\author{
Arnon Sturm \\ Technion - Israel Institute of Technology \\ Haifa 32000, ISRAEL \\ sturm@tx.technion.ac.il
}

\begin{abstract}
To develop agent-based systems, one needs a methodology that supports the development process as common in other disciplines. In recent years, several such methodologies and modeling techniques have been suggested. An important question is, to what extent do the existing methodologies address the developers' needs. In this paper we attempt to answer this question. In particular, we discuss suitability of agent modeling techniques to agent-based systems development. In evaluating existing modeling techniques, we address criteria from software engineering as well as characteristics of agent-based systems. Our evaluation shows that some aspects of modeling techniques for agent-based systems may benefit from further enhancements. As we show, these aspects include distribution, concurrency, testing and communication richness. We also find space for (relatively small) improvements in aspects such as the refining of the models throughout the development process and the coverage and consistency checking of the suggested models.
\end{abstract}

\section{INTRODUCTION}

The discipline of Agent Oriented Software Engineering (AOSE) has emerged during the last decade. In this domain, methodologies and modeling techniques have been suggested in order to support the development process of agent-based systems. Both the scientific and industrial communities have recognized the potential advantages of agent-based systems. Nevertheless, the number of deployed commercial agent-based applications is not large. One of the reasons for this is the lack of mature, off the shelf, methodologies for agentbased application development. The need for such methodologies has been discussed by several studies $[1,7,9]$. For example, according to [6], AOSE is a key factor for introducing agent-based systems to the industry as an engineering approach. In addition, one would like the advantages of an organized development process such as reusability, testing, and maintenance to be applied to agentbased systems as well. As part of AOSE research we can find methodologies and modeling techniques that present concepts of software agent modeling at different levels, i.e., different lifecycle stages. In this paper we examine these techniques in attempt to answer the following questions: (1) Which agent-based system characteristics and software engineering principles are addressed within AOSE modeling techniques, and to what extent? (2) What

Permission to make digital or hard copies of all or part of this work for personal or classroom use is granted without fee provided that copies are not made or distributed for profit or commercial advantage and that copies bear this notice and the full citation on the first page. To copy otherwise, or republish, to post on servers or to redistribute to lists, requires prior specific permission and/or a fee.

AGENTS'01, February 11-13, 2001, Montréal, Quebec, Canada.

Copyright 2001 ACM 1-58113-326-X/01/0005 ...\$5.00 should be the properties of the future agent-oriented modeling techniques? The previous work mentioned above overlooked some of the software engineering aspects and agent application properties. Moreover, that work did not provide evaluation criteria for assessing advantages and drawbacks of different modeling techniques. In this paper we provide answers to the first question. Our on-going research attempts to provide answers to the second question as well. Here, we review some agent-oriented modeling techniques and evaluate them according to both software engineering criteria and agent-based system characteristics. From this evaluation we draw conclusions regarding the extent to which the examined agent modeling techniques address developers' needs. In addition, we examine the need for additional modeling features and extensions. Our evaluation and conclusions are based on available documents regarding those techniques. Note that some documents and tools of these techniques are not publicly available.

The paper is organized as follows. In section 1, we present the criteria we use to examine the agent-based systems modeling techniques. In section 2, a case study describes a working project dealing with an auction agent. This case study is used to demonstrate the modeling techniques we evaluate. Section 3 describes the modeling techniques and their evaluation. Section 4 summarizes the evaluation and presents the conclusions.

\subsection{Software Engineering Evaluation Criteria}

The major goal of this paper is to evaluate existing modeling technique for agent-based system. For this, one should refer to software-engineering criteria and agent-based system characteristics. In this section we focus on the former, whereas the latter will be discussed in the next section. There are numerous criteria for evaluating the quality of a modeling technique from the softwareengineering viewpoint. Our selection of software-engineering criteria for the evaluation is based on $[1,12]$ and their relevancy to agent-based systems. Following these criteria helps in providing documentation, encouraging reuse and maintaining the development lifecycle. Among other desirable criteria, a modeling technique should adhere to the following:

1. Preciseness: the semantics of a modeling technique must be unambiguous in order to avoid misinterpretation of the models (of the modeling technique) by those who use it.

2. Accessibility: a modeling technique should be comprehensible to both experts and novices.

3. Expressiveness (and applicable to multiple domains): a modeling technique should be able to present:

- $\quad$ the structure of the system;

- $\quad$ the knowledge encapsulated within the system;

- $\quad$ the data flow within the system;

- the control flow within the system;

- the interaction of the system with external systems. 
4. Modularity: a modeling technique should be expressible in stages. That is, when new specification requirements are added, there is no need to modify pervious parts, and these may be used as part of the new specification.

5. Complexity Management: a modeling technique should be expressed, and then examined, at various levels of detail. Sometimes, high-level requirements are needed, while in other situations, more detail is required. Examination and development of all levels should be facilitated.

6. Executability (and testability): either a prototyping capacity or a simulation capacity should be associated with at least some aspects of the modeling technique. That is, the modeling technique has related tools that allow (possibly inefficient) computation for sample input. These should demonstrate possible behaviors of the system being modeled, and help developers determine whether the intended requirements have been expressed.

7. Refinability (and implementability): a modeling technique should provide a clear path for refining a model through gradual stages to reach an implementation, or at least for clearly connecting the implementation level to the design specification.

8. Analyzability: a methodology, or, preferably, an associated tool is available to check the internal consistency or implications of the models, or to identify aspects that seem to be unclear, such as the interrelations among seemingly unrelated operations. Such tools encourage both consistency and coverage.

9. Openness: a modeling technique should provide a good basis for modeling agent-based systems without coupling them to a specific architecture, infrastructure or programming language.

\subsection{Agent-Based Systems Characteristics}

As mentioned above, another facet of evaluating agent-based system modeling technique is the examination of agent-based system characteristics. In this section we focus on selected agentcharacterizing features as a basis for our evaluation. We select the agent-based system characteristics from $[7,8,9,17]$.

1. Autonomy: unlike objects, agents may be active and are responsible for their own activities: the agent has control over both its reactive and proactive behaviors. The modeling technique should support the capability of describing an agent's self-control feature.

2. Complexity: agent-based systems are basically sets of components (agents) that interact with each other in order to achieve their goals. These systems may consist of decision making mechanisms, learning mechanisms, reasoning mechanism and other complex algorithms. Modeling complex algorithms and mechanisms requires strong expressive power and many layers of details. A modeling technique should support such expressiveness in order to model the functionality of agent-based systems. Moreover, the complexity feature requires that modeling technique should be modular, support complexity management and describe the complex nature of an agent.

3. Adaptability: agent-based systems have to be flexible in order to adjust their activities to the dynamic environmental changes. The adaptability feature may require that a modeling technique be modular and that it can activate each component according to the environmental state.
4. Concurrency: an agent may need to perform several activities/tasks at the same time. The concurrency feature raises the requirement that some agent-based systems must be designed as parallel processing systems. This requires ability to express parallelism and concurrency in the design and implementation/deployment stages.

5. Distribution: multi-agent systems are sometimes working on different hosts and should be distributed over a network. This requires ability to express distribution in the design and implementation/deployment stages.

6. Communication richness: a basic definition of an agent consists of its autonomous activity. As such, the agent must establish communication with its environment. The environment may include other agents and information sources. The communication is characterized by its type (either interagent communication or intra-agent communication), its content and its architecture (e.g. client-server, peer-to-peer). This requires that a modeling technique should be able to express the communication characterization in order to produce agent communication command or sentences during the implementation stage.

\section{A CASE STUDY AUCTION AGENT}

The modeling techniques evaluated in this paper are demonstrated via a case study of an existing, fully implemented, single agent application. In this section we describe the agent, however its use as a case study only appears in the proceeding sections. This agent is an auction agent, that is, it participates and bids in web-based auctions on behalf of its user. To be able to use this agent for purchasing a specific item, its user must provide the agent with the following parameters: item number, user identification, private maximal price, bid step, monitoring frequency and bidding strategy and its parameters. Once activated, the agent enters the auction site and locates the specific product. Then, the agent monitors the site and retrieves the following information: leading offer (current price, user), bid step and closing date. After retrieving this information the agent parses it and acts according to the strategy that was previously selected by the user. The agent halts its auction-related activity either when its buying startegy dictate withdrawal or when the closing date of the auction passes. As mentioned above, the agent we deal with in our case study was fully developed, that is, analysis, design, implementation and testing were all performed.

The agent software components are the following:

1. The configurator is a GUI component that enables the agent's user to control and monitor the agent's activity.

2. The parser translates the information retrieved from the auction site into an internal structure.

3. The bidder submits bids to the auction site according to the agent's buying strategy. It implements two stages of the bidding (as required by the specific auction site): the bid and its confirmation.

4. The manager controls the agent's activity, monitors the auction site, activates the parser, determines the next bid as needed, activates the bidder as required and terminates the agent's purchasing activity.

In our case studies throughout the paper we refer to both the agent described above and the auction site with which the agent was coupled. In times, we find it necessary to provide some details of the auction site, since, to study and evaluate the modeling techniques 
presented in this paper, the environment in which the agent resides is important too.

\section{AGENT MODELING TECHNIQUES}

In this section, we describe and evaluate several agent modeling techniques. For each modeling technique we provide a short description, a case study (based on our auction agent), and evaluation based on the criteria mentioned above.

\subsection{AOM and GAIA}

Agent-Oriented Methodology (AOM) and GAIA, which extends $\mathrm{AOM}$, are methodologies that concentrate on the modeling aspect of agent-based systems. Our analysis and evaluation are based on AOM and GAIA details as appear in [19,20]. Following, the term AOM will refer to GAIA as well. AOM is based on a set of models, which are used in different stages of the agent development (both analysis and design). Following the AOM guidelines, the analysis of an intended agent-based system results in the definition of roles, which are characterized by three attributes - permissions, responsibilities and protocols - and an interaction model which depicts interactions between roles:

- The permissions attribute states what resources may be used to carry out the role and what resource constraints the role's executor is subject to.

- The responsibilities attribute determines the functionality of the role. This functionality is expressed in terms of safety and liveness properties. The safety properties indicate what conditions must be true through all of the states of the system. The safety properties are invariant. The liveness properties indicate what sequence of states the role's lifecycle consists of, and what alternative computational paths are available for the role.

- The protocols attribute states the interactions of the role with other roles. In addition it states the internal activities of the role. AOM distinguishes between external activities (refered to as protocols) and internal ones. The internal activities will be elaborated upon as a part of the service model later in this section.

Each role has a schema, which describes the permissions, responsibilities and protocols attributes. An example of role schemata is depicted in Figure 1. In this Figure, the Manager's role schema (of the case study Auction Agent) consists of a description of the way in which the Manager should control the agent's activities. The Manager's protocols and activities consist of the CheckAuctionSite protocol, the ActivateParser activity, the CheckForBid activity and the Bid protocol. The Manager's permission is comprised of (a) read access ${ }^{1}$ to the AuctionDetails (which is a part of the internal structure of the agent); (b) supplying of the ItemNumber. The responsibilities are separated into two categories: safety and liveness.

The true statement that appears at the safety properties entry indicates that there are no constraints on the role. As for the liveness properties, the statement indicates that the order of execution of the protocols and activities is as following: after the protocol of CheckAuctionSite is executed the ActivateParser activity is performed. Following, the CheckForBid activity is

${ }^{1}$ In AOM notation, bolded words are reserved words which are being used for indicating the access right to a specific resource. performed. This sequence of protocols and activities occurs one or more times. After that sequence, the Bid Protocol may occur ${ }^{2}$. The interaction model is used for a protocol description.

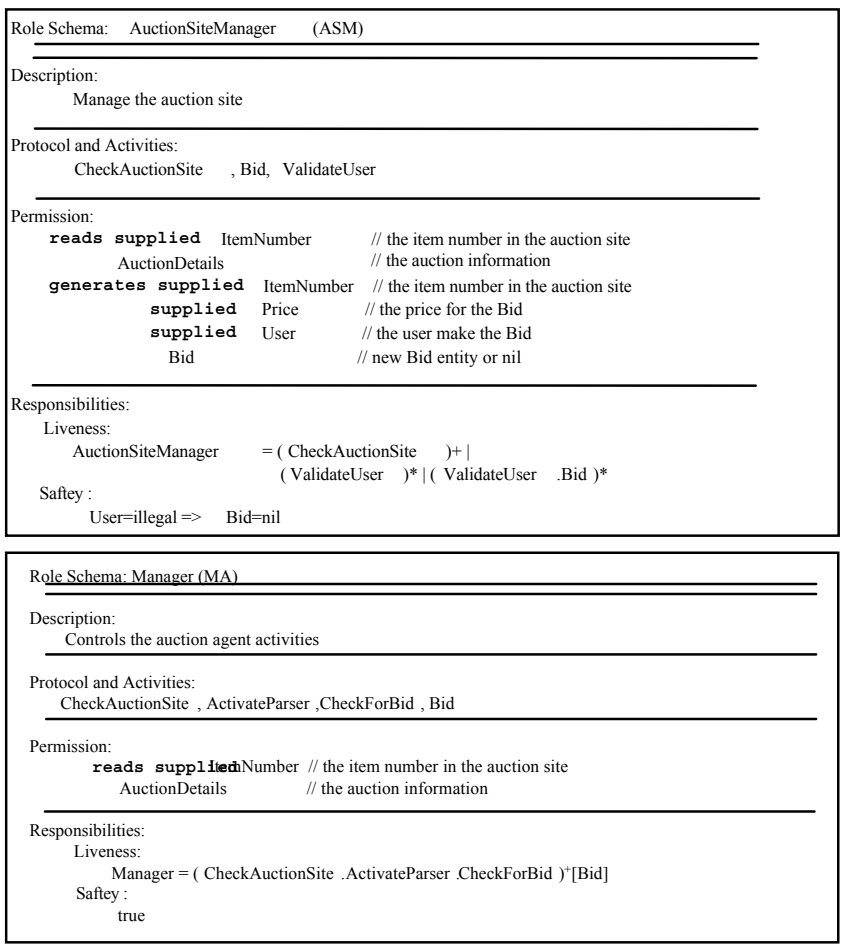

Figure 1: the Manager and the AuctionSiteManager schemata

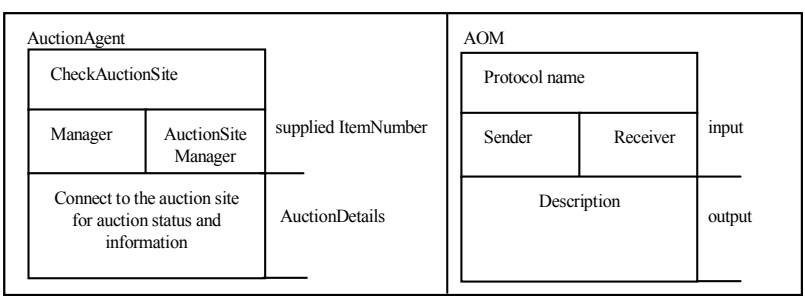

Figure 2: The Interaction Model of the CheckAuctionSite protocol

An example of the interaction model is depicted in Figure 2. The Figure shows the generic interaction model of AOM (on the right side) and the case study model (on the left side). In the case study model, there is a description of the CheckAuctionSite protocol, which consists of the roles of Mangaer and the AuctionSiteManager. The Mangaer supplies the ItemNumber and the AuctionSiteManager returns the AuctionDetails.

The AOM design phase consists of three models. These models are derived from the role and the interaction models built in the analysis stage:

- The agent model defines roles, which are carried out by each agent type. Each agent type has an indication of its instances number. The agent model is depicted in Figure 3. The Figure shows the roles assigned to the agent types and the agent types instances number.

\footnotetext{
${ }^{2}$ The square brackets indicate an optional activity or protocol.
} 


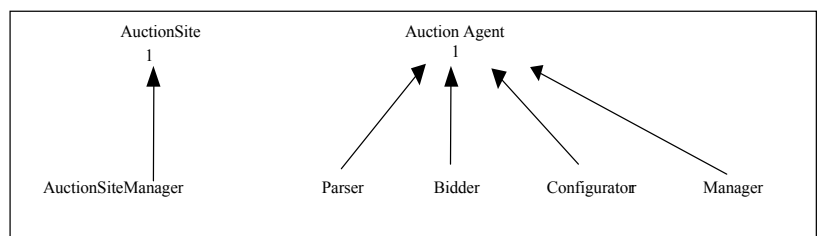

Figure 3: The Agent model represents the Auction Agent

- $\quad$ The service model describes the functionality of the agent by extending the protocols and responsibilities models from the analysis stage.Each service includes the documentation of its input, output, pre-condition and post-condition. The service model is depicted in Figure 4. It presents a subset of the agent's services. These are derived from the Manager, the AuctionSiteManager and the interaction model.

\begin{tabular}{|c|c|c|c|c|}
\hline Service & Input & Output & $\begin{array}{l}\text { Pre } \\
\text { condition }\end{array}$ & $\begin{array}{l}\text { Post } \\
\text { Condition } \\
\end{array}$ \\
\hline $\begin{array}{l}\text { get auction } \\
\text { details }\end{array}$ & ItemNumber & AuctionDetails & true & True \\
\hline Validate user & User & Exist & true & $\begin{array}{l}\text { (exist=true) } \vee \\
\text { (exist=false) }\end{array}$ \\
\hline Bid & $\begin{array}{l}\text { User, } \\
\text { ItemNumber, } \\
\text { Price }\end{array}$ & Success & user exist & $\begin{array}{l}\text { (success=true) } \\
\vee(\text { success }=\text { false })\end{array}$ \\
\hline
\end{tabular}

Figure 4: The Service Model

- $\quad$ The acquaintance model describes the communication path between the agent types. This is graphically expressed by a directed graph as depicted in Figure 5. The nodes in the graph represent agent types whereas the vertices represent the communication path. In the case study acquaintance model, there is a bi-directonal communication path between the AuctionSite and the AuctionAgent.

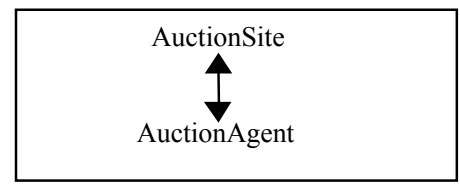

Figure 5: The Acquaintance Mode

\subsubsection{Software-Engineering Evaluation}

1. Preciseness: the liveness and safety properties, which are used for depicting the functionality of a role in a formal way (i.e., for each symbol and notation there is a clear meaning and interpretation), makes AOM accurate and prevents misinterpretation of the modeled functionality. The symbols and notations of each of the other AOM models have a clear meaning as well.

2. Accessibility: AOM is easy to understand and use due to its simple models and their clarity. Understanding and usage of AOM can be achieved by following the description and examples of the different models as appear in [19,20].

3. Expressiveness: $\mathrm{AOM}$ is expressive and can handle a large variety of systems due to its generic structure. However, AOM is mostly suitable for small and medium scale systems. This is because of its flatness, which limits the ability to model large amount of details. The structure of the system, the encapsulated knowledge, the data and control flows are not presented explicitly within AOM.

4. Modularity: AOM is mostly modular due to its design with some building blocks such as roles, protocols, activities and agent types. In AOM, one can assign new roles to agents and remove ones with no effect on the internal model of the roles. However, changes within the protocol might cause changes within the internal structure of the role. These result in changes in permissions of the role, thus limits the modularity of AOM.

5. Complexity Management: in AOM, there is no hierarchical presentation or any other mechanism for complexity management. The system description is flat.

6. Executability: this issue is not dealt with within AOM.

7. Refinability: this issue is not dealt with within AOM.

8. Analyzability: this issue is not dealt with within AOM.

9. Openness: AOM does not dictate any particular architecture, infrastructure or programming language. It leaves the implementation issue open for the programmer to deal with. Thus, it has an open modeling technique.

\subsubsection{Agent-based System Characteristics}

1. Autonomy: in AOM the autonomy is expressed by the fact that the role encapsulates its functionality (i.e., it is responsible for it). This functionality is internal and is not affected by the environment, thus represents the role's autonomy. In addition, in AOM one can model alternative computational paths, which gives the role (and agents that consist of this role) the autonomy in making decisions.

2. Complexity: in AOM one can describe the agent's complexity by integrating several models: the role model, the acquaintance model and the agent model. However, it is difficult to model some procedural processes and complex computation in AOM.

3. Adaptability: adaptability in AOM can be expressed by the liveness properties. The liveness property operators within AOM symbolize optional execution, thus expresses the potential adaptability of the agent to environmental changes (although the changes are not described explicitly).

4. Concurrency: this issue is not dealt with within AOM.

5. Distribution: AOM allows the expression of distribution via the acquaintance model, where each role can be performed on any host or processor. Yet, it does not explicitly address the distribution issue.

6. Communication richness: the communication aspects that are dealt with within AOM are the protocols and the communication paths between agents. However, the content of a message and the communication architecture are not expressed in any of the AOM models.

\subsection{ADEPT}

The Advanced Decision Environment for Process Tasks (ADEPT) system is a complete infrastructure for designing and implementing multi-agent systems. As such, ADEPT supplies a set of tools (i.e., models and a language) to achieve its purpose. Our evaluation of ADEPT is based on details provided in $[8,10,11,16]$. In ADEPT, the main building block (from the modeling viewpoint) is the agency. An agency is recursively defined and consists of a single responsible (or controlling) agent, a set of tasks and a set of sub-agencies. The tasks that the agent is responsible for can be viewed as simple services with a well-defined input, output, guard and functional specifications. An ADEPT-specific language called SDL (Service Description Language) defines the services and the information of the agent. In addition, ADEPT has a negotiation model, which includes a protocol, a service level agreement (SLA) and a reasoning 
model. The protocols are relatively standard and are based on speech-act theory. To communicate, agents in ADEPT need a protocol and a SLA. The latter is a template that defines the agreement type. One of the fields of the SLA indicates the service associated with it. This template should be known to both the client and the server agents. The reasoning model consists of two knowledge-based components: a declarative one and a procedural one. The declarative one sets the negotiation context and the procedural one specifies which action should be taken given the declarative knowledge. The procedural knowledge-base is represented as a set of strategies and mechanisms for selecting between them.

Figure 6 depicts an ADEPT model of the case study Auction Agent. The Figure presents two agents associated with their agencies and tasks. The Auction Agent is responsible for the agency, which should perform the tasks of managing, bidding, configuring and parsing. A rounded rectangle indicates a responsible agent, a box indicates an agency, diamond denotes a task and the arrows indicate the services supplied by the agent. The specification of the information encapsulated within the agents is depicted in Figure 7 and the service specification of the Get_Auction_Details service is depicted in Figure 8.

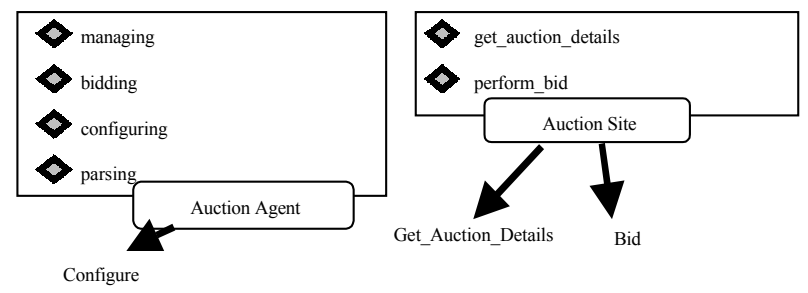

Figure 6: The Agent-Agencies Model

\begin{tabular}{|l|l|}
\hline (class Agent_Info & $\begin{array}{l}\text { (class Auction_Site_Info } \\
\text { (Types_String item_number) } \\
\text { (Types_String item_number) } \\
\text { (Typer_User user) } \\
\text { (Types_Float private_maximal_price) } \\
\text { (Types_Float bid_step) } \\
\text { (Types_Long monitoring_frequency) } \\
\text { (Types_Strategy bidding_strategy)) } \\
\text { (Types_Bid leading_offer) }\end{array}$ \\
\hline
\end{tabular}

Figure 7: SDL description of an information object

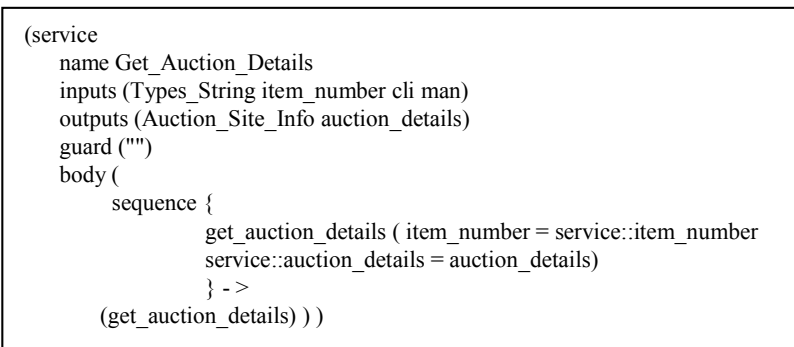

Figure 8: SDL description of the Get_Auction_Details service provided by the Auction Site agent

\subsubsection{Software-Engineering Evaluation}

1. Preciseness: a formal specification of SDL is provided. Other formal specifications of ADEPT have not been published yet however exist. These formal specifications prevent misinterpretation of ADEPT models.

2. Accessibility: learning, understanding and implementing ADEPT models is not difficult. The majority of the ADEPT models are intelligible and provide a clear notations and semantics. However, the reasoning model might introduce some difficulties in its understanding and assimilation, due to its complex logic.

3. Expressiveness: integration of the models supported by ADEPT provides an expressive power, especially for business processes. These models represent the structure of the system (sometimes a reflection of the organization structure), the behavior of the system and its response to environmental changes. The data and control flow can be analyzed and understood from the SDL and the reasoning model, but they are not presented explicitly. This implies difficulty for the designers and implementers in modeling and understanding the data and control flow. In addition, SDL represents the knowledge structure within the system. An implementation of an ADEPT agent or MAS is coupled to the ADEPT architecture, which means that its uses the ADEPT modules (such as Service Execution Module and Communication Module). These modules execute the different models mentioned before. Hence, there is no need for defining the system structure.

4. Modularity: the use of agencies and agents increases the modularity, as agencies can be easily moved from one agency to another.

5. Complexity Management: hierarchical presentation and complexity management can be achieved by using the agent and agencies model, which enables (de-) composition of agents and agencies.

6. Executability: ADEPT has its own engine, which means that once the modeling phase was finished the agent can be executed. However, it is not clear how much effort the designer has to invest in the modeling phase (analysis and design) in order to get the agent to run.

7. Refinability: ADEPT has its own definition language (SDL), which defines the services derived from the model of agents and agencies. This means that the model of agents and agencies can be refined by SDL.

8. Analyzability the consistency and coverage checks are not dealt with within ADEPT.

9. Openness: ADEPT is a closed system. The different models are tightly coupled to the ADEPT architecture.

\subsubsection{Agent-based System Characteristics}

1. Autonomy: the autonomous nature of an agent is modeled by encapsulating tasks within an agency. This means that agent that controls the agency is autonomous in the timing and execution of the encapsulated tasks. The decision-making aspect of the agent's autonomy is modeled by combining the service model and the reasoning model within ADEPT. These models describe the agent's activities upon environment and the agent's state changes.

2. Complexity: by using the ADEPT models one can present large and complex systems. However, the tools supplied by ADEPT are not powerful enough to express complex algorithms.

3. Adaptability: the adaptability in ADEPT is expressed by the reasoning model. The reasoning model has the capability of representing several states regarding the negotiation context and additional rules. The negotiation context and additional 
rules provide the agent with guidance regarding the activities it should perform. This enables the agent to adapt to environmental and state changes.

4. Concurrency: is not dealt with within ADEPT from the modeling viewpoint.

5. Distribution: is not dealt with within the ADEPT from the modeling viewpoint.

6. Communication richness: the communication between agents is handled by the system architecture, but its modeling is unclear.

\subsection{DESIRE}

The Design and Specification of Interacting Reasoning (DESIRE) framework is a complete environment for design and implementation of MAS. It allows the system designer to explicitly and precisely specify both the intra-agent and inter-agent functionality. Our evaluation of DESIRE is based on the information provided in $[3,4,13,15]$. In DESIRE, the following models are supported: (1) task (de-) composition, (2) information exchange, (3) sequencing of (sub-) tasks, (4) subtask delegation and (5) knowledge structure.

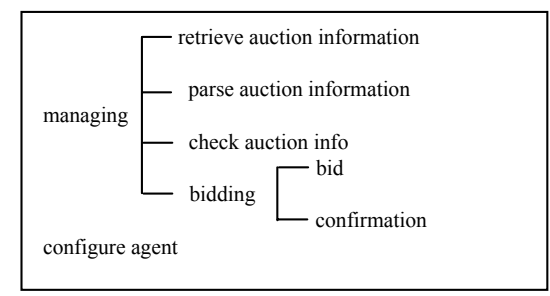

Figure 9: Task Hierarchy of the Auction Agent

The task (de-) composition model includes the information about the task hierarchy, the task input, the task output and the relationships between tasks. Each task in the hierarchy can be primitive as well as composed. Additional information regarding the task composition model is encapsulated within the information exchange model. The task hierarchy model of the Auction Agent is depicted in Figure 9. In this Figure, the managing task consists of the retrieve auction information, parse auction information, check auction info and bidding tasks. The bidding task consists of the bid and confirmation tasks. The tasks which do not consist of other tasks are primitive.

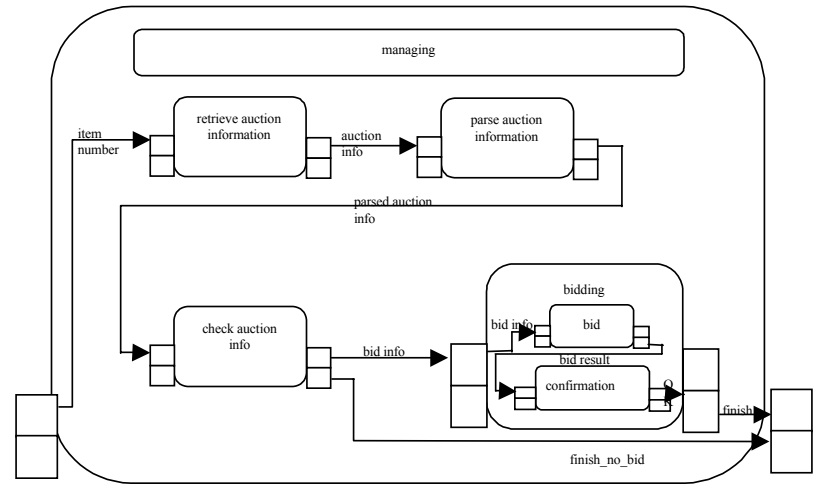

Figure 10: The component managing of the Auction Agent

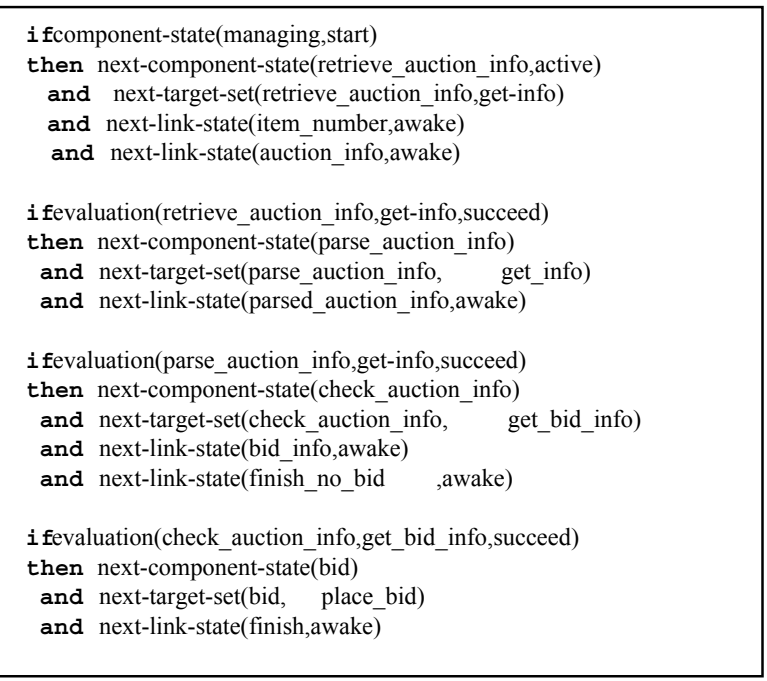

Figure 11: The task control knowledge of managing

In DESIRE, the information exchange between tasks is specified as information links between components. Each information link directs the output of one component to the input of another one. The information exchange model is depicted in Figure 10. This Figure shows the task hierarchy that was previously presented in Figure 9. The additional information is the information links that depict the data flow. For example, the link ItemNumber indicates that there is an input to the retrieve auction information task. Its output is fed as input, via the auction info link to the check auction info task. Note that the information exchange model has a formal syntax. Task sequencing is explicitly modeled within the component as task control knowledge, which can be seen in Figure 11. This knowledge includes the order of subtasks, their activation target (usually referred to as a goal in the context of agents) and the amount of effort, which can be afforded. The target is the focus of the activity of the component and the effort is the component and the link states. For example, if the task parse_auction_information succeeds then the next task should be check_auction info and its target should be get_bid_info. In addition, DESIRE awakes the relevant links that should be activated if the check_auction_info task is successful. DESIRE has the additional advantage that the specifications and their semantics can be expressed formally, using temporal logic.

\subsubsection{Software-Engineering Evaluation}

1. Preciseness: the DESIRE infrastructure is based on temporal logic, which is precise and prevents misinterpretation of the modeling outcomes.

2. Accessibility: DESIRE has a wide range of modeling capabilities. However, this richness results in difficulty to learn and implement it.

3. Expressiveness: DESIRE can express many application domains using and integrating its models. DESIRE has many tested systems such as Generic Design Agent [5] and Collaborative Information Agents [13]. The data flow modeling within DESIRE is performed using the links in the task composition model. The control flow can be understood from the task control knowledge. The data modeling is done using the knowledge structure mechanism. An implementation of a DESIRE agent or MAS is coupled to the DESIRE architecture, 
which means that it uses the DESIRE infrastructure. This infrastructure supports the execution of the modeled system. Hence, there is no need for defining the system structure.

4. Modularity: DESIRE supports modularity within the component model. This means that components can be changed and replaced without any effect on others.

5. Complexity Management: the hierarchical presentation and complexity management is achieved within the models of task hierarchy and components.

6. Executability: DESIRE has its own engine. After the modeling is finished, the modeled system should run. DESIRE has an automated implementation generator, however, the designer can not determine the implemented software architecture.

7. Refinability: the DESIRE models are not formally assigned to a specific development stage (i.e., analysis, design, and implementation). However, DESIRE enables to refine the models at any stage.

8. Analyzability: correctness and coverage are checked using the formal semantic specification.

9. Openness: DESIRE is an open system in the sense that it is not coupled to a specific programming language or architecture.

\subsubsection{Agent-based System Characteristics}

1. Autonomy: the autonomy of an agent can be expressed within the DESIRE task control knowledge. In this model, one can track the possible execution paths of the agent activities regardless of the environment. That is, the agent has its autonomy of executing the tasks. Also, one can model a decision mechanism of the agent, which reflects the autonomy of the agent from the decision-making aspect.

2. Complexity: DESIRE models enable one to describe the required complexity of a software agent. However, (according to the reviewed literature) DESIRE does not provide tools for modeling complex computation.

3. Adaptability: the flexibility of the agent is modeled within the task control knowledge. In this model the agent's behavior can change according to new goals and other environmental changes.

4. Concurrency: the concurrency issue is dealt with from the functional point of view but not from the execution point of view. This means that one can model concurrency within the task control knowledge but the implementation of this concurrency is not explicitly addressed.

5. Distribution: the distribution issue in DESIRE can be expressed using the task composition model, where each task can be performed on any host or processor. However the distribution issue is not addressed explicitly in DESIRE.

6. Communication richness: the communication in DESIRE is partially dealt with by presenting the links between components. However, the DESIRE literature does not refer to a message content and means.

\section{CONCLUSION}

In this paper, we reviewed a few of the existing techniques for agentbased system modeling. In addition to these techniques, we have also performed evaluation of the Formal Agent Framework [14] and the Agent Unified Modeling Language [2]. The details of that evaluation are presented in [18]. In this review we have used widely acceptable software-engineering and agent-based system evaluation criteria. The goal of this paper is not to identify the best modeling technique for agent-based systems among the existing ones, but to point out the issues that should be addressed in future research and development of agent-oriented modeling techniques. As a secondary result, we have also provided a set of criteria that developers of agent-based systems can use to determine the appropriate modeling technique and system for a specific project according to its characterization.

Table 1. Modeling Techniques Evaluation Summary

\begin{tabular}{|l|c|c|c|}
\hline \multicolumn{1}{|c|}{$\begin{array}{c}\text { Methods } \\
\text { Criteria }\end{array}$} & AOM & ADEPT & DESIRE \\
\hline Preciseness & $*$ & $*$ & + \\
\hline Accessibility & + & - & $*$ \\
\hline Expressiveness & $*$ & + & + \\
\hline Modularity & - & + & + \\
\hline $\begin{array}{l}\text { Complexity } \\
\text { Management }\end{array}$ & - & + & + \\
\hline Executability & NS & + & $*$ \\
\hline Refinablility & NS & $*$ & $*$ \\
\hline Analyzability & NS & NS & + \\
\hline Openness & + & - & + \\
\hline Autonomy & $*$ & + & + \\
\hline Complexity & - & $*$ & $*$ \\
\hline Adaptability & $*$ & $*$ & + \\
\hline Concurrency & NS & NS & $*$ \\
\hline Distribution & NS & NS & NS \\
\hline $\begin{array}{l}\text { Communication } \\
\text { richness }\end{array}$ & - & - & - \\
\hline+ good, $*$ satisfying, - dissatisfying, NS - not supported \\
\hline
\end{tabular}

Table 1 provides a qualitative summary of our evaluation. Each modeling technique is evaluated by indicating to what level it addresses each criterion. In general, we found the modeling techniques adequately addressing agent-based system characteristics. Yet, our conclusions regarding software engineering issues suggest that AOSE still has a way to go to provide industrially applicable modeling technique. In particular, the following issues may benefit from further enhancements:

- $\quad$ Executability - AOM does not deal with the execution issues. ADEPT deals with the implementation issues of the execution, i.e. a prototype can be created out of the design specification. DESIRE generates working applications out of the design specification (although these applications are not necessarily efficient). All of the examined modeling techniques do not address the testing issue. Testing is an important part of the software development process since it allows increased reliability of the final product. As such, it would be beneficial to have an automatic code and test cases generation included in agent-oriented modeling techniques.

- $\quad$ Refinablility - The modeling techniques that we reviewed do not clearly define the development process. The models, which are part of the modeling technique, are not associated with the development stages (i.e., analysis, design and implementation). This results in difficulty to determine what part of the agent should be modeled in each development stage. Hence, it is not clear how the refinement should be done and what level of detail should be produced at each stage of the development lifecycle. In addition, each one of the modeling techniques uses models of which encapsulated 
information (sometimes) overlaps, and the designer may need to synchronize between them.

- $\quad$ Analyzablility - the reviewed modeling techniques do not have accessible tools to perform the modeling phase (although some of them do have working environments). However, most of them have a firm basis for the consistency and coverage checks.

- Openness - the evaluation of openness indicates that most of the modeling techniques that deal with the execution issue are not based on a specific architecture for MAS.

Although agent-based system characteristics are usually properly addressed, a few issues can benefits from being dealt with:

- $\quad$ Distribution - the distribution and concurrency issues are not dealt with from the deployment viewpoint in ADEPT and DESIRE and in AOM it is not addressed at all.

- Communication richness - the reviewed modeling techniques provide some details regarding the required communication. However, they do not model the message content as well as the communication architecture.

To conclude, the current agent-oriented modeling techniques already provide a wide array of features advantageous for agent modeling. However, as our evaluation suggests, there is a need for further exploration of the issues mentioned above. Base on these findings, we intend in future research, to address the needs of agent-based system developers. This should be done in order to find the required modeling techniques and components for building agent-based systems.

\section{REFERENCES}

[1] M. A. Ardis, J. A. Chaves, L. J. Jagadeesan, P. Mataga, C. Puchol, M. G. Staskauskas and J. Von Olnhausen, A Framework for Evaluating Specification Methods for Reactive Systems, Experience Report, Proc. of 17th Intl. Conf. on Software Engineering, 1995.

[2] B. Bauer, J.P. Muller, and J. Odell, Agent UML: A Formalism for Specifying Multiagent Software Systems: P. Ciancarinin and M.J. Wooldridge (eds.) Agent-Oriented Software Engineering, LNCS 1957, Springer, 2000, pp. 91-103.

[3] F. M. T. Brazier, B. Dunin-Keplicz, N. R. Jennings and J. Treur, DESIRE: Modelling Multi-Agent Systems in a Compositional Formal Framework. Intl. Journal of Cooperative Information Systems, vol. 6, Formal Methods in Cooperative Information Systems: Multi-Agent Systems, (M. Huhns and M. Singh, eds.), 1997, pp. 67-94

[4] F. M. T. Brazier, B. Dunin-Keplicz, J. Treur and L. C. Verbrugge, Modeling Internal Dynamic Behaviour of BDI agents. In: J.-J. Ch. Meyer and P.Y. Schobbes (eds.), Formal Models of Agents, LNAI 1760, Springer, 1999, pp. 36-56.

[5] F. M. T. Brazier., C. M. Jonker, J. Treur and N.J.E. Wijngaards, Compositional Design of a Generic Design Agent. In: G. Luger, L. Interrante (eds.), Proc. of AAAI Workshop on AI and Manufacturing: State of the Art and State of Practice. AAAI Press, 1998, pp. 30-39.
[6] C. A. Iglesias, M. Garijo, and J. C. Gonzalez. A survey of agent-oriented methodologies. In J. P. Muller, M. P.Singh, and A. S. Roa, (eds.), Intelligent Agent V, Proc. of ATAL-98, LNAI 1555, Springer, 1999,pp. 317-330.

[7] N. R. Jennings and M. Wooldridge, Agent-Oriented Software Engineering, Handbook of Agent Technology (ed. J. Bradshaw) AAAI/MIT Press, 2000 (to appear).

[8] N. R. Jennings, P. Faratin, M. J. Johnson, P. O'Brien, M. E. Wiegand: Using Intelligent Agents to Manage Business Processes, Proc. of PAAM96, London, UK, 1996, 345-360

[9] N. R. Jennings, On Agent-Based Software Engineering, Artificial Intelligence, 117 (2), 2000 , 277-296.

[10] N. R. Jennings, P. Faratin, T. J. Norman, P. O'Brien and B. Odgers, Autonomous Agents for Business Process Management, Int. Journal of Applied AI 14 (2), 2000, 145-189.

[11] N. R. Jennings, P. Faratin, T. J. Norman, P. O'Brien, B. Odgers and J. L. Alty, Implementing a Business Process Management System using ADEPT: A Real-World Case Study, Intl. Journal of Applied AI 14 (5), 2000, 421-465.

[12] S. Katz, Draft of Formal Specification Method Book, http://www.cs.technion.ac.il/ cs236368/, 2000.

[13] C. M. Jonker, M. Klusch, and J. Treur, Design of Collaborative Information Agents. In: M. Klusch, and L. Kerschberg (eds.), Cooperative Information Agents IV, Proc. of CIA 2000. LANI 1860, Springer, 2000, pp. 262-283.

[14] M. Luck and M. d'Inverno, Structuring a Z Specification to Provide a Formal Framework for Autonomous Agent Systems, Proc. of ZUM'95, J. Bowen and M. Hinchey (eds.), LNCS, 967, 47-62, Springer, 1995.

[15] M. Mulder, J. Treur and M.Fisher, Agent Modelling in MetateM and DESIRE. In: M. P. Singh, A. S. Rao, M. J. Wooldridge (eds.), Intelligent Agents IV, Proc. of ATAL'97. LNAI 1365, Springer, 1998, pp. 193-207.

[16] T. J. Norman, N. R. Jennings, P. Faratin and E. H. Mamdani: Designing and implementing a multi-agent architecture for business process management, in Intelligent Agents III (eds. J. P. Mueller, M. J. Wooldridge and N. R. Jennings) LNAI 1193, Springer, 1996, 261-275.

[17] O. Shehory, Architectural Properties of Multi-Agent Systems, Technical Report CMU-RI-TR-98-28, The Robotics Institute, Carnegie Mellon University, 1998.

[18] O. Shehory and A. Sturm, Evaluating Agent-Based System Modeling Techniques, Technical Report TR-ISE/IE-003-2000, Faculty of Industrial Engineering and Management Technion Israel Institute of Technology, 2000.

[19] M. Wooldridge, N. R. Jennings, and D. Kinny, A Methodology for Agent-Oriented Analysis and Design Proc. of Agents-99, Seattle, WA, 1999, 69-76.

[20] M. Wooldridge, N. R. Jennings, and D. Kinny, The Gaia Methodology for Agent-Oriented Analysis and Design, Journal of Autonomous Agents and MAS 3 (3), 2000, 285-312. 